\title{
Local Configurations of Digital Hyperplanes
}

\author{
Yan Gérard \\ gerard@llaic.u-clermont1.fr \\ Laboratoire de Logique et d'Informatique de Clermont1 (LLAIC1) \\ IUT, Département d'Informatique, Ensemble Universitaire des Cézeaux \\ B.P. 86, 63172 Aubière Cedex, France
}

\begin{abstract}
The aim of this article is to provide some arithmetical tools in order to study the local properties of digital hyperplanes.

With the help of the new general notion of configuration, we investigate the arrangement of the different combinatorial structures contained in a digital hyperplane. The regularity of this deployment is controlled by two arithmetical functions that we call $\operatorname{code}_{(I)}$ and $\operatorname{boundary}_{(I)}$. By using these two simple tools, we prove that the local configurations in a functional digital hyperplane only depends on its normal vector and that their number is less than the size of the chosen neighborhood.

Keywords: windows, local configurations, digital hyperplanes.
\end{abstract}

\section{Introduction}

The fact that such classical mathematical tools as differentiability are totally ineffective in digital geometry has aroused the curiosity of more than one author especially in DGCI conferences. Some of the mathematical tools designed to study the digital functions mapping $\mathbb{Z}^{2}$ to $\mathbb{Z}$ include the notions of tricubes introduced by Isabelle DEBLED-RENESSON [CV97|D95 RY97S97, of $n$-m local configurations [R91|V99] or under another name the $n$-m-cubes. They are both built on the same principle: one looks at the functions through a window that we translate and which is rectangular and $3 \times 3$ in size for the tricubes or $m \times n$ in size for the $m-n$ local configurations. But why should we only consider rectangular windows? We could as well look at a function through a triangular window or even through any window and that is the idea we are going to develop here. In this paper, we take any window and in order to justify this choice, we will generalize the theorem of J-P REVEILLÈs where he proves that a naive plane contains at most $n m$ different $n-m$ cubes R95 V99. In fact, this result is a direct consequence of a general analysis inspired by the integer part function. The keypoint is that we reduce these multidimensional combinatorial problems to unidimensional questions by using a fundamental lemma called trampoline and two functions called $\operatorname{code}_{(I)}$ and $\operatorname{boundary}_{(I)}$. Finally, we will illustrate the use of these tools by sketching a proof of the well-known fact that there exist exactly 40 tricubes. 


\section{Local configurations}

The purpose of this section is to introduce the general notion of a local configuration of a map from a group into a group. At first, we introduce a definition for the windows of a group.

Definition 2.1 - - Let $\mathrm{G}$ be a group, a finite subset of $\mathrm{G}$ containing the neutral element is called a window of $\mathrm{G}$. The cardinality of the subset is called the size of the window.

The subset $\{0 ; 3.65 ; 4.5 ;-6\}$ is an example of window of the additive group $\mathbb{R}$ of size 4 . In this paper, the windows are denoted $W$, their size $s$ and we denote their elements $w^{1}, w^{2}, \ldots, w^{i}, \ldots, w^{s}$ so that the neutral element of the window is always the first on the list $\left(w^{1}=0_{G}\right.$, neutral element of $\left.G\right)$. The indices of the elements of the window are written as exponents so that we will be able to denote $w_{j}^{i}$ the coordinates of $w^{i}$ in the case where the group $G$ is $\mathbb{R}^{n}$ or $\mathbb{Z}^{n}$.

Now, let $G$ and $G^{\prime}$ be two groups, $W=\left\{w^{1}=0, w^{2}, \cdots, w^{s}\right\}$ a window of $G$ of size $s$ and $f$ a function mapping $G$ to $G^{\prime}$. We define the local configurations through $W$ of the function $f$.

Definition 2.2 .- The local configuration through $\mathrm{W}$ of the function $\mathrm{f}$ at a point $\mathrm{x}$ belonging to $\mathrm{G}$ is the element of $\left(\mathrm{G}^{\prime}\right)^{\mathrm{s}}$ :

$$
\left(f\left(x+w^{1}\right)-f(x), f\left(x+w^{2}\right)-f(x), \cdots, f\left(x+w^{s}\right)-f(x)\right) .
$$

As $w^{1}=0$, the first value $f\left(x+w^{1}\right)-f(x)$ of the $s$-uple is always the neutral of $G^{\prime}$.

If the function maps $\mathbb{Z}^{n-1}$ to $\mathbb{Z}$, this notion can be intended for use in computing something like the normal direction of digital objects (a classical problem in imagery). For the investigation of such maps, we will be inspired by the local configurations through a window $W$ of a classical map: the integer part function.

\section{The integer part function}

We recall that the integer part function []$: \mathbb{R} \rightarrow \mathbb{Z}$ is defined by $[x]=\max \{y \in$ $\mathbb{Z} \mid y \leq x\}$. We denote also $\{x\}$ the fractional part of $x$ such that $x=[x]+\{x\}$ or again with a different notation $\{x\}=x \bmod 1$. The integer part function is a map sending the additive group $\mathbb{R}$ to the additive group $\mathbb{Z}$. We study it because it performs a transition between continuous and digital worlds. We are going to investigate its local configurations through a window $W$ by using a simple lemma.

\subsection{Jump-lemma}

Let $x$ and $w$ be two real numbers.

Lemma 3.1 . - (a preliminary version of jump-lemma)- We have $[\mathrm{x}+\mathrm{w}]-[\mathrm{x}]=[\mathrm{w}]$ if $\{\mathrm{x}\}<1-\{\mathrm{w}\}$

$$
=[\mathrm{w}]+1 \text { otherwise. }
$$


The proof uses nothing else than inequalities. The consequence of this lemma is that for a given real $w$, the difference $[x+w]-[x]$ can only take two different values: $[w]$ or $[w+1]$. The lemma specifies when the difference takes one or other possible value : it depends on the comparison of $\{x\}$ with $1-\{w\}$. To represent these two "separated" values, we will introduce some new notations:

Definition 3.1 - - We define the maps code $: \mathbb{R} \rightarrow[0,1[$ by $\operatorname{code}(\mathrm{x})=\{\mathrm{x}\}$ and boundary $: \mathbb{R} \longrightarrow] 0,1]$ by boundary $(\mathrm{w})=1-\{\mathrm{w}\}$.

These two functions are closely related to the functions boundary $(I)$ and $\operatorname{code}_{(I)}$ that we will use later in order to investigate the local configurations of digital hyperplanes. With these notations, jump-lemma can be written out:

Lemma 3.2 .-(Jump-lemma)- We have

$[\mathrm{x}+\mathrm{w}]-[\mathrm{x}]=[\mathrm{w}]$ if $\operatorname{code}(\mathrm{x})<\operatorname{boundary}(\mathrm{w})$

$=[\mathrm{w}]+1$ otherwise.

For a given real $w$ and any real $x$, the value taken by $[x+w]-[x]$ only depends on the comparison of the value code $(x)$ with the value boundary $(w)$ and it is the keypoint in our investigations into the local configurations of the integer part function.

\subsection{The local configurations of the integer part function}

Let $\left(c^{i}\right)_{1 \leq i \leq s}$ be the local configuration of the integer part function at a point $x$ through a given window $W=\left\{w^{1}, w^{2}, \cdots, w^{s}\right\}$ of $\mathbb{R}$. By definition, the value $c^{i}$ is $\left[x+w^{i}\right]-[x]$ and we could compute it directly. According to the jump-lemma, $c^{i}$ is equal to $\left[w^{i}\right]$ if $\operatorname{code}(x)<\operatorname{boundary}\left(w^{i}\right)$ and to $\left[w^{i}\right]+1$ otherwise. It shows that the local configuration at point $x$ only depends on the values $\left[w^{i}\right]$ and on the comparison of code $(x)$ with the $s$ values boundary $\left(w^{i}\right)$. These $s$ numbers boundary $\left(w^{i}\right)$ can be sorted and so ordered, they partition the intervall $[0,1[$ into $s$ regions which are closed on the left and opened on the right side (when two boundaries are equal, the region that they surround is empty). To sum up, we can say that for a given window $W$, the local configuration at a point $x$ through the window $W$ only depends on the region where there is $\operatorname{code}(x)$. Then each region corresponds to a local configuration and the local configuration at $x$ is the local configuration associated with the region of $\operatorname{code}(x)$. We will illustrate this argument by an example.

Example.- Let us describe the local configurations of the function integer part through the window of reals $W=\left\{w^{1}=0 ; w^{2}=5,6 ; w^{3}=2,45 ; w^{4}=3,75\right.$; $\left.w^{5}=-1,8\right\}$. By definition, the local configuration of the integer part function at the point $x$ through $W$ is

$$
([x+0]-[x] ;[x+5,6]-[x] ;[x+2,45]-[x] ;[x+3,75]-[x] ;[x-1,8]-[x]) .
$$

The boundaries associated with the window $W$ are the values boundary $\left(w^{1}\right)=1$; boundary $\left(w^{2}\right)=0,4 ; \operatorname{boundary}\left(w^{3}\right)=0,55 ; \operatorname{boundary}\left(w^{4}\right)=0,25$; 
boundary $\left(w^{5}\right)=0,8$. After being sorted, they partition the intervall $[0,1[$ into regions (fig 1).

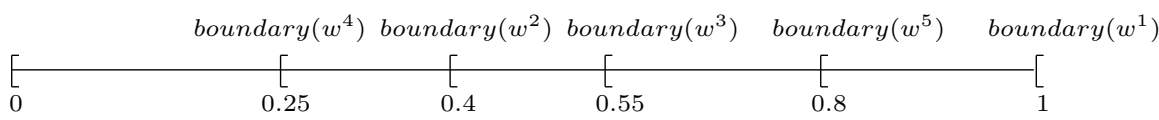

Figure 1.- The partition of $[0,1$ [ into regions according to the 5 values boundary $\left(w^{i}\right)$.

If for example $\operatorname{code}(x)$ belongs to the region between the boundaries boundary $\left(w^{3}\right)$ and boundary $\left(w^{5}\right)$, then we have $\operatorname{code}(x)<$ boundary $\left(w^{1}\right)$ which implies, according to the jump-lemma, $\left[x+w^{1}\right]-[x]=\left[w^{1}\right]=0$

$\operatorname{code}(x) \geq$ boundary $\left(w^{2}\right)$ which implies $\left[x+w^{2}\right]-[x]=\left[w^{2}\right]+1=6$ $\operatorname{code}(x) \geq$ boundary $\left(w^{3}\right)$ which implies $\left[x+w^{3}\right]-[x]=\left[w^{3}\right]+1=3$ $\operatorname{code}(x) \geq \operatorname{boundary}\left(w^{4}\right)$ which implies $\left[x+w^{4}\right]-[x]=\left[w^{4}\right]+1=4$ and code $(x)<$ boundary $\left(w^{5}\right)$ which implies $\left[x+w^{5}\right]-[x]=\left[w^{5}\right]=-2$. Thus the region lying between the boundaries boundary $\left(w^{3}\right)$ and boundary $\left(w^{5}\right)$ corresponds to the local configuration $(0,6,3,4,-2)$. According to the jumplemma, each region corresponds in the same way to a local configuration (fig 2).

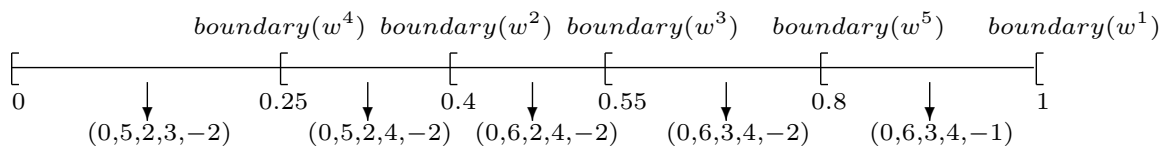

Figure 2.- The regions and their corresponding local configurations

The local configuration at a point $x$ is the one which corresponds to the region of $\operatorname{code}(x)$. At $x=4,33$ for example, $\operatorname{code}(x)=0,33$ and we conclude that at 4,33 , the local configuration through $W$ is $(0,5,2,4,-2)$.

From a mathematical point of view, since our window has size $s$, there are at most $s$ different regions and then the number of different local configurations is bounded by $s$. It follows that the integer part function cannot have more than $s$ different local configurations through a given window of size $s$. Now, we are going to use the same way of thinking to investigate the local configurations of digital hyperplanes.

\section{The local configurations of a digital functional hyperplane}

Let $P$ be any functional in $x_{n}$ digital hyperplane defined by the double-inequality

(I) $\quad h \leq a_{1} x_{1}+a_{2} x_{2}+\cdots+a_{i} x_{i}+\cdots+a_{n} x_{n}<h+\left|a_{n}\right|$

where $h \in \mathbb{R},\left(a_{1}, \ldots, a_{i}, \ldots, a_{n}\right) \in \mathbb{R}^{n}$ with $a_{n}$ different from 0 and where the variable $x=\left(x_{1}, \ldots, x_{i}, \ldots, x_{n}\right)$ belongs to $\mathbb{Z}^{n}$. The coefficients $a_{i}$ are not 
supposed rational and it is also the definition used in [V99. In [R91] on the other hand, the numbers $a_{i}$ are only taken in $\mathbb{Q}$. The functional digital hyperplane $P$ is the graph of a map $p: \mathbb{Z}^{n-1} \longrightarrow \mathbb{Z}$. If the coefficient $a_{n}$ is positive, $p(x)=$ $-\left[\left(\sum_{i=1}^{n-1} a_{i} x_{i}-h\right) / a_{n}\right]$. If $a_{n}$ is negative, $p(x)=\left[\left(h-\sum_{i=1}^{n-1} a_{i} x_{i}\right) / a_{n}\right]$. Then we have

$$
p(x)=\epsilon\left[\left(\sum_{i=1}^{n-1} a_{i} x_{i}-h\right) /\left|a_{n}\right|\right]
$$

where $\epsilon=-1$ if $a_{n}$ is positive and $\epsilon=+1$ if $a_{n}$ is negative. In order to investigate the local configurations of the function $p$, we need a lemma inspired by the investigation of the integer part function.

\subsection{Trampoline-lemma}

This term "trampoline" refers to the fact that this lemma is both a consequence and a multidimensional generalization of jump-lemma. Before writing it out, we will introduce the functions $\operatorname{code}_{(I)}(x)$ and $\operatorname{boundary}_{(I)}(w)$ :

Definition 4.1 .- We call code $_{(\mathrm{I})}$ the function

$$
\begin{gathered}
\operatorname{code}_{(\mathrm{I})}: \mathbb{Z}^{\mathrm{n}-1} \longrightarrow\left[0,\left|\mathrm{a}_{\mathrm{n}}\right|[\right. \\
\mathrm{x}=\left(\mathrm{x}_{1}, \mathrm{x}_{2}, \cdots \mathrm{x}_{\mathrm{n}-1}\right) \longmapsto \operatorname{code}_{(\mathrm{I})}(\mathrm{x})=\left(\sum_{\mathrm{i}=1}^{\mathrm{n}-1} \mathrm{a}_{\mathrm{i}} \mathrm{x}_{\mathrm{i}}-\mathrm{h}\right) \bmod \left|\mathrm{a}_{\mathrm{n}}\right|
\end{gathered}
$$

and boundary(I) the function

$$
\begin{gathered}
\left.\left.\operatorname{boundary}_{(\mathrm{I})}: \mathbb{Z}^{\mathrm{n}-1} \longrightarrow\right] 0,\left|\mathrm{a}_{\mathrm{n}}\right|\right] \\
\mathrm{w}=\left(\mathrm{w}_{1}, \mathrm{w}_{2}, \cdots \mathrm{w}_{\mathrm{n}-1}\right) \longmapsto \operatorname{boundary}_{(\mathrm{I})}(\mathrm{w})=\left|\mathrm{a}_{\mathrm{n}}\right|-\left(\left(\sum_{\mathrm{i}=1}^{\mathrm{n}-1} \mathrm{a}_{\mathrm{i}} \mathrm{w}_{\mathrm{i}}\right) \bmod \left|\mathrm{a}_{\mathrm{n}}\right|\right) .
\end{gathered}
$$

With these two functions, we can establish a lemma of jump similar to the jump-lemma except that the functions are no more [ ], code and boundary but $p, \operatorname{code}_{(I)}$ and boundary $(I)$ :

Lemma 4.1.-(trampoline-lemma)-Let $x$ and $w$ be two elements of $\mathbb{Z}^{n-1}$, we have $\mathrm{p}(\mathrm{x}+\mathrm{w})-\mathrm{p}(\mathrm{x})=\epsilon\left[\left(\sum_{\mathrm{i}=1}^{\mathrm{n}-1} \mathrm{a}_{\mathrm{i}} \mathrm{w}_{\mathrm{i}}\right) /\left|\mathrm{a}_{\mathrm{n}}\right|\right]$ if $\operatorname{code}_{(\mathrm{I})}(\mathrm{x})<\operatorname{boundary}_{(\mathrm{I})}(\mathrm{w})$

$$
=\epsilon\left[\left(\sum_{\mathrm{i}=1}^{\mathrm{n}-1} \mathrm{a}_{\mathrm{i}} \mathrm{w}_{\mathrm{i}}\right) /\left|\mathrm{a}_{\mathrm{n}}\right|\right]+\epsilon \text { otherwise. }
$$

Proof. We can prove trampoline-lemma in a more general framework. If we examine the function $p(x)$, it appears as the restriction to $\mathbb{Z}^{n-1}$ of a function $q(x)$ of the form $k[a . x-l]$ where $k$ and $l$ are some real numbers, where $a \in \mathbb{R}^{n-1}$ and where the variable $x$ is taken in $\mathbb{R}^{n-1}$. We have $q(x+w)-q(x)=k([(a . x-$ $l)+a . w]-[a . x-l])$ and according to the jump-lemma, this difference is equal to $k[a . w]$ if $\{a . x-l\}<1-\{a . w\}$ and to $k[a . w]+k$ otherwise. Then we have here a 
multi-dimensional generalization of jump-lemma. For the particular case which interests us, $(a . x-l) \bmod 1$ is $\left(\left(\sum_{i=1}^{n-1} a_{i} x_{i}-h\right) /\left|a_{n}\right|\right) \bmod 1$ and $1-(a . w \bmod 1)$ is $1-\left(\left(\sum_{i=1}^{n-1} a_{i} w_{i} /\left|a_{n}\right|\right) \bmod 1\right)$. Instead of working with these values, we multiply them by $\left|a_{n}\right|$ so that they would be integer values if $\left(a_{i}\right)_{1 \leq i \leq n-1} \in \mathbb{Z}^{n-1}$. Thus we obtain the functions $\operatorname{code}_{(I)}$, boundary $_{(I)}$ and the lemma.

Remark. - Trampoline-lemma is fundamental because the investigation of the differences $p(x+w)-p(x)$ (where $x$ and $w$ are in $\mathbb{Z}^{n-1}$ ) which is a multidimensional combinatorial problem is reduced to unidimensional "reflections". Thus the two functions $\operatorname{code}_{(I)}$ and boundary $_{(I)}$ defined from a double-inequality (I) come to light as two powerful arithmetical tools for the study of the local properties of digital hyperplanes.

\subsection{The local configurations of the function $p$}

Let $W=\left\{w^{1}=0, w^{2}, \cdots, w^{s}\right\}$ be a window of $\mathbb{Z}^{n-1}$ and $\left(c^{i}\right)_{1 \leq i \leq s}$ the local configuration of the function $p$ through $W$ at a point $x$. By definition, the value $c^{i}$ is $p\left(x+w^{i}\right)-p(x)$ and we could compute it directly. According to the trampolinelemma, $c^{i}$ is equal to $\epsilon\left[\left(\sum_{i=1}^{n-1} a_{i} w_{i}\right) /\left|a_{n}\right|\right]$ if $\operatorname{code}_{(I)}(x)<\operatorname{boundary}_{(I)}\left(w^{i}\right)$ and to $\epsilon\left[\left(\sum_{i=1}^{n-1} a_{i} w_{i}\right) /\left|a_{n}\right|\right]+\epsilon$ otherwise. It shows that the local configuration at point $x$ only depends on the values $\epsilon\left[\left(\sum_{i=1}^{n-1} a_{i} w_{i}\right) /\left|a_{n}\right|\right]$ and on the comparison of $\operatorname{code}_{(I)}(x)$ with the $s$ values boundary $(I)\left(w^{i}\right)$. These $s$ numbers boundary $y_{(I)}\left(w^{i}\right)$ called the boundaries can be sorted and this is an essential point. So ordered, they partition the intervall $\left[0,\left|a_{n}\right|[\right.$ into $s$ regions which are closed on the left and opened on the right side (when two boundaries are equal, the region that they surround is empty). To sum up, we can say that for a fixed function $p$ and a fixed window $W$, the local configuration of $p$ through $W$ at a point $x$ only depends on the region where there is $\operatorname{code}_{(I)}(x)$. Then each region corresponds to a local configuration and the local configuration at $x$ is the local configuration associated with the region of $\operatorname{code}_{(I)}(x)$. We will illustrate this analysis by an example.

Example.- Let $P$ be the digital plane functional in $x_{3}$ and characterized by the double-inequality

$$
\text { (I) } \quad-2 \leq 3 x_{1}+4 x_{2}+7 x_{3}<5
$$

The digital plane $P$ is the graph of the function $p: \mathbb{Z}^{2} \longrightarrow \mathbb{Z}$ defined by $p\left(x_{1}, x_{2}\right)=-\left[\left(3 x_{1}+4 x_{2}+2\right) / 7\right]$. Let $W$ be the window of $\mathbb{Z}^{2}$ of size $s=5$ where $w^{1}=(0,0), w^{2}=(-2,-1), w^{3}=(1,0), w^{4}=(2,0)$ and $w^{5}=(2,1)$ (fig 3$)$.

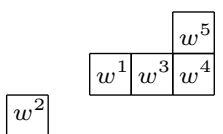

Figure 3.- The window $W$ 
The local configuration of the function $p$ at a point $x \in \mathbb{Z}^{2}$ is the element of $\mathbb{Z}^{5}$ defined by $\left(p\left(x+w^{1}\right)-p(x) ; p\left(x+w^{2}\right)-p(x) ; p\left(x+w^{3}\right)-p(x) ; p\left(x+w^{4}\right)-p(x)\right.$; $\left.p\left(x+w^{5}\right)-p(x)\right)$. The previous arithmetical tools are the functions $\operatorname{code}_{(I)}: \mathbb{Z}^{2} \longrightarrow\left[0,7\left[\right.\right.$ defined by $\operatorname{code}_{(I)}\left(x_{1}, x_{2}\right)=\left(3 x_{1}+4 x_{2}+2\right) \bmod 7$ and boundary $\left.\left._{(I)}: \mathbb{Z}^{2} \longrightarrow\right] 0,7\right]$ defined by boundary $(I)\left(w_{1}, w_{2}\right)=7-\left(\left(3 w_{1}+\right.\right.$ $\left.\left.4 w_{2}\right) \bmod 7\right)$. The boundaries associated with the window $W$ are the values $\operatorname{boundary}_{(I)}\left(w^{1}\right)=7$, boundary $_{(I)}\left(w^{2}\right)=3$, boundary $_{(I)}\left(w^{3}\right)=4$, boundary $_{(I)}\left(w^{4}\right)=1$ and boundary $(I)\left(w^{5}\right)=4$. After being sorted, they partition the interval $[0,7[$ into regions (fig 4 ).

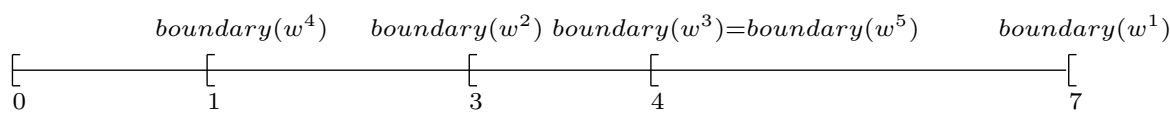

Figure 4.- The partition of $[0,7$ [ into regions according to the 5 values $\operatorname{boundary}_{(I)}\left(w^{i}\right)$

If for example, $\operatorname{code}_{(I)}(x)$ belongs to the region between $\operatorname{boundary}_{(I)}\left(w^{4}\right)=1$ and $\operatorname{boundary}_{(I)}\left(w^{2}\right)=3$, then we have:

$\operatorname{code}_{(I)}(x)<\operatorname{boundary}_{(I)}\left(w^{1}\right)=7$ which implies according to the trampolinelemma $p\left(x+w^{1}\right)-p(x)=\epsilon\left[\left(\sum_{i=1}^{2} a_{i} \cdot w_{i}^{1}\right) /\left|a_{3}\right|\right]=-[(0.3-0.4) / 7]=0$; $\operatorname{code}_{(I)}(x)<$ boundary $_{(I)}\left(w^{2}\right)$ which implies

$$
p\left(x+w^{2}\right)-p(x)=\epsilon\left[\left(\sum_{i=1}^{2} a_{i} . w_{i}^{2}\right) /\left|a_{3}\right|\right]=-[(-2.3-1.4) / 7]=2 ;
$$

$\operatorname{code}_{(I)}(x)<\operatorname{boundary}_{(I)}\left(w^{3}\right)$ which implies

$$
p\left(x+w^{3}\right)-p(x)=\epsilon\left[\left(\sum_{i=1}^{2} a_{i} . w_{i}^{3}\right) /\left|a_{3}\right|\right]=-[(1.3+0.4) / 7]=0 ;
$$

$\operatorname{code}_{(I)}(x) \geq \operatorname{boundary}_{(I)}\left(w^{4}\right)$ which implies

$$
p\left(x+w^{4}\right)-p(x)=\epsilon\left[\left(\sum_{i=1}^{2} a_{i} . w_{i}^{4}\right) /\left|a_{3}\right|\right]+\epsilon=-[(2.3+0.4) / 7]-1=-1 ;
$$

$\operatorname{code}_{(I)}(x)<\operatorname{boundary}_{(I)}\left(w^{5}\right)$ which implies

$$
p\left(x+w^{5}\right)-p(x)=\epsilon\left[\left(\sum_{i=1}^{2} a_{i} \cdot w_{i}^{5}\right) /\left|a_{3}\right|\right]=-[(2.3+1.4) / 7]=-1 .
$$

Then the region $\left[\right.$ boundary $_{(I)}\left(w^{4}\right)$, boundary $_{(I)}\left(w^{2}\right)$ [ corresponds to the local configuration drawn fig 5 .

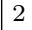

$$
\begin{array}{|l|l|l|}
\cline { 2 - 3 } \multicolumn{2}{l|}{} & -1 \\
\hline 0 & 0 & -1 \\
\hline
\end{array}
$$

Figure 5.- The local configuration associated to the region [boundary $\left(w^{4}\right)$, boundary $\left(w^{2}\right)[$

In the same way, the different regions correspond to the local configurations drawn fig 6 . 


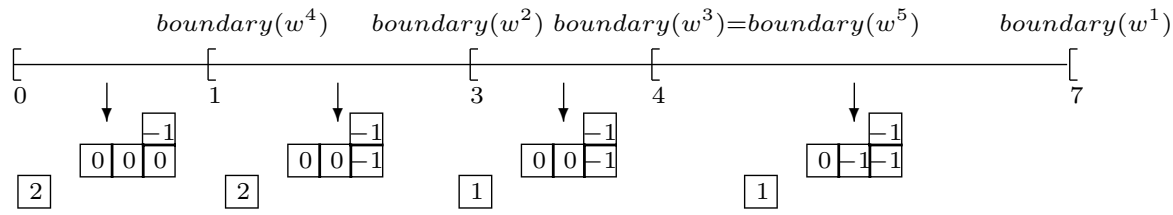

Figure 6 .- The regions and their corresponding local configurations.

The local configuration at $x$ is the one which corresponds to the region of $\operatorname{code}_{(I)}(x)$. At $\left(x_{1}, x_{2}\right)=(-3,5)$ for example, we have code $(I)(-3,5)=6$ and it follows that at $(-3,5)$ the local configuration is $(0,1,-1,-1,-2)$ (fig 6$)$.

This analysis provides the arrangement of the local configurations in a digital hyperplane. In particular, we have partitioned the interval $\left[0,\left|a_{n}\right|[\right.$ into at most $s$ disjoined regions. From this it follows that the function $p$ cannot have more than $s$ different local configurations through a given window of size $s$. It provides the following theorem:

Theorem 4.1 - A functional digital hyperplane has at most s different local configurations through a given window of size s.

This theorem generalizes to all windows and all dimensions a result already known in dimension $n=3$ for the rectangular windows R95 V99. Some other results concerning symmetric local configurations can be deduced from the analysis we have developed in this part.

\section{Applications}

By using the functions $\operatorname{code}_{(I)}$ and boundary $_{(I)}$, we can prove the following theorem:

Theorem 5.1 .-(direction-theorem)- Two digital hyperplanes $\mathrm{P}$ and $\mathrm{P}^{\prime}$ of respective double-inequalities $\mathrm{h} \leq \sum_{\mathrm{i}=1}^{\mathrm{n}} \mathrm{a}_{\mathrm{i}} \mathrm{x}_{\mathrm{i}}<\mathrm{h}+\left|\mathrm{a}_{\mathrm{n}}\right|$ and $\mathrm{h}^{\prime} \leq \sum_{\mathrm{i}=1}^{\mathrm{n}} \mathrm{a}_{\mathrm{i}} \mathrm{x}_{\mathrm{i}}<$ $\mathrm{h}^{\prime}+\left|\mathrm{a}_{\mathrm{n}}\right|$ have the same set of local configurations through a given window.

This theorem is trivial for the digital hyperplanes which have a rational normal direction because they are image one from the other by a translation. It holds in the general case for which such translations do not necessarily exist because the windows are by definition finite. If one considered infinite "windows", the theorem would be false. The proof comes from a lemma of presence, which says that a local configuration is obtained if and only if it corresponds to a non-empty region.

This theorem is useful because it allows the normal directions or in other words the normal cone of a given local configuration to be defined and the FourierMotzkin algorithm can be used in order to compute it [FST96] (for rectangular windows, we can also use [D95]). 
The functions $\operatorname{code}_{(I)}$ and boundary $(I)$ can be used to obtain several results and we are going to illustrate their use by sketching a proof to the well-known fact that there exist exactly 40 tricubes (enumeration already obtained from several algorithms [D95|FST96|S97]). Let us remind the reader that the tricubes are the local configurations of the functions $p(x, y)=-[(a x+b y-h) / c]$ with $0<a<b<c$ (functions from the digital planes functional in $z$ of doubleinequalities (I) $h \leq a x+b y+c z<h+c)$ through the square window $\left\{w^{1}=\right.$ $(0,0), w^{2}=(-1,-1), w^{3}=(0,-1), w^{4}=(1,-1), w^{5}=(-1,0)$, $\left.w^{6}=(1,0), w^{7}=(-1,1), w^{8}=(0,1), w^{9}=(1,1)\right\}$ of sides 3 by 3 .

Firstly, instead of working directly with the whole domain $0<a<b<c$, we suppose $0<a<b<a+b<c$. With $a, b, c$ taken in this more restrictive domain, the values $-\left[\left(a . w_{x}^{i}+b . w_{y}^{i}\right) / c\right]$ which appear in trampoline-lemma for $p\left(x+w^{i}\right)-p(x)$ are determined. Then we know that, $p\left(x+w^{1}\right)-p(x)$ is always null, the values $p\left(x+w^{2}\right)-p(x), p\left(x+w^{3}\right)-p(x), p\left(x+w^{4}\right)-p(x), p\left(x+w^{5}\right)-p(x)$ are equal to 1 or 0 and the values $p\left(x+w^{6}\right)-p(x), p\left(x+w^{7}\right)-p(x)$, $p\left(x+w^{8}\right)-p(x), \quad p\left(x+w^{9}\right)-p(x)$ are equal to 0 or -1 . We have also $\operatorname{boundary}_{(I)}\left(w^{1}\right)=c$, boundary $_{(I)}\left(w^{2}\right)=a+b, \operatorname{boundary}_{(I)}\left(w^{3}\right)=b$, $\operatorname{boundary}_{(I)}\left(w^{4}\right)=b-a, \operatorname{boundary}_{(I)}\left(w^{5}\right)=a, \operatorname{boundary}_{(I)}\left(w^{6}\right)=c-a$,

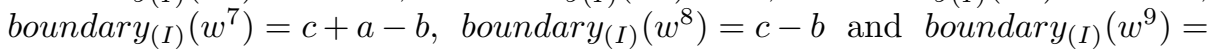
$c-a-b$. Now, for a fixed value $c$, we draw the domain of $(a, b) \in \mathbb{R}^{2}$ verifying $0<a<b<a+b<c$ and the straight lines of equations boundary $\left(w^{i}\right)=$ boundary $\left(w^{j}\right)$ (fig 7$)$. On one side of such a straight line, we have boundary $\left(w^{i}\right)>$ $\operatorname{boundary}\left(w^{j}\right)$ and on the other side, we have boundary $\left(w^{i}\right)<\operatorname{boundary}\left(w^{j}\right)$.

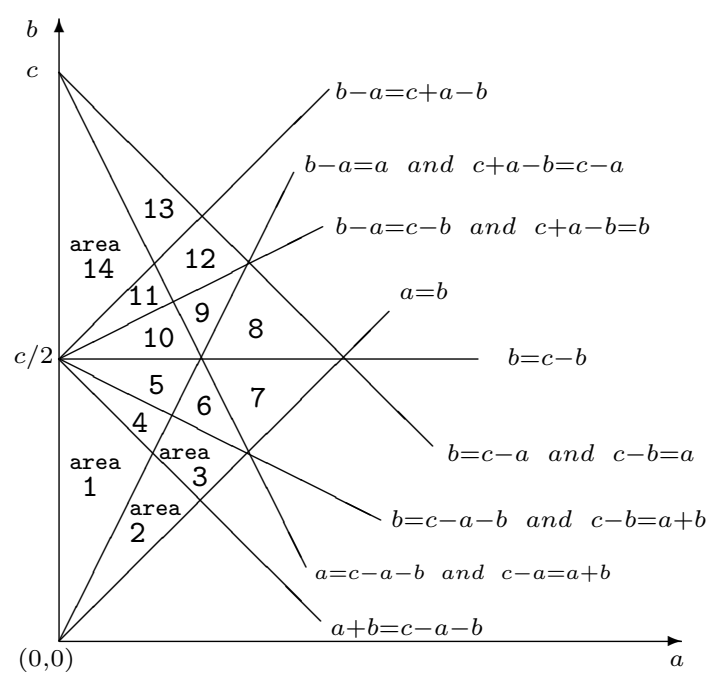

Figure 7 .- the domain $0<a<b<a+b<c$ cut into areas

The areas of the domain $0<a<b<a+b<c$ so cut up correspond each one to an order of the boundaries. As we have seen that the values $-\left[\left(a . w_{x}^{i}+b . w_{y}^{i}\right) / c\right]$ 
were constant in all the domain $0<a<b<a+b<c$, each area and its corresponding order of the boundaries gives 9 local configurations (fig 8).
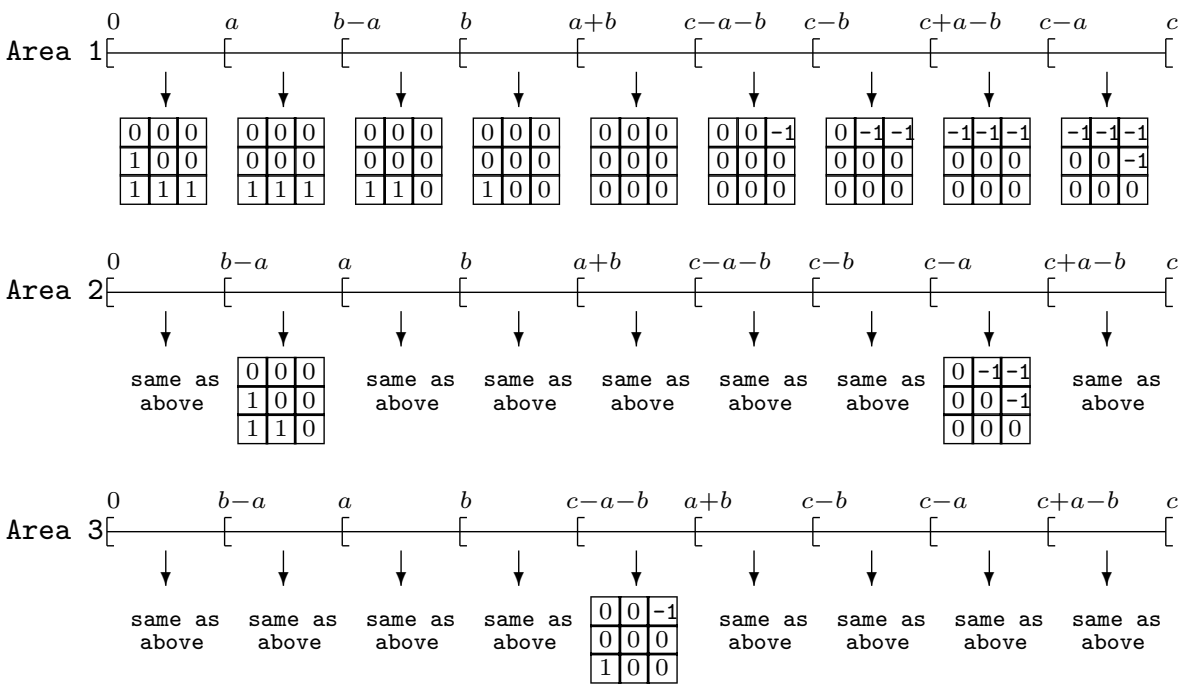

Figure 8 - - The orders of the values boundary $(I)\left(w^{i}\right)$ in the areas $1,2,3$ and the associated tricubes.

A presence-lemma ensures that any digital plane with its coefficients $a, b, c$ in the interior of an area takes the 9 corresponding local configurations and one concludes that those are always 9 tricubes. Thus, this construction provides all the tricubes of the domain $0<a<b<a+b<c$. If we then study the domain $0<a<b<c \leq a+b$ in the same way, we obtain the 40 tricubes shared out in the figure already presented in [CV97].

\section{Conclusion}

In this paper, the main idea is to look at digital hyperplanes through windows of any shape. Instead of directly tackling the problem of the local configurations of the functional digital hyperplanes, we have investigated the local configurations of the integer part function. Their study does not require any other tools than a lemma (jump-lemma) and two functions (code and boundary). In order to tackle our initial problem, namely the local configurations of the functional digital hyperplanes, we can generalize the lemma (trampoline-lemma) and the two functions. In fact, in this multidimensional framework, the situation is exactly the same as for the integer part function except that the maps are no longer [ ], code and boundary but $p\left(x_{1}, x_{2} \cdots x_{n}\right)=\epsilon\left[\left(\sum_{i=1}^{n-1} a_{i} x_{i}-h\right) /\left|a_{n}\right|\right]$, $\operatorname{code}_{(I)}$ and boundary $_{(I)}$. These arithmetical tools allow us to compute the local configurations of a functional digital hyperplane and describe their deployment. They can be used to prove many theoretical results as we have seen with the 40 
tricubes. Generally speaking one can say that they permit us to go easily from a double-inequality of a digital hyperplane to the local configurations.

\section{Acknowledgements}

Acknowledgements are due to Denis RicharD, Jean-Pierre ReveILLÈs and Carol FYNN.

\section{References}

CV97. J.M. Chassery \& J. Vittone, Coexistence of Tricubes in Digital Naive Plane. 7th Conference on Discrete Geometry in Computer Imagery, Montpellier 1997.

D95. I. Debled-Renesson, Étude et reconnaissance des droites et plans discrets. Thèse de doctorat soutenue à l'Université Louis Pasteur de Strasbourg, 1995.

F96. J. FrançON, Sur la topologie d'un plan arithmétique. Theorical Computer Sciences 156, 1996.

FST96. J. FrançOn, J.M. SChramm \& M. TAJine, Recognizing arithmetic straight lines and planes. 6th Conference on Discrete Geometry in Computer Imagery, Lyon, 1996.

R91. J.P. REVEILLÈs, Géométrie discrète, calcul en nombre entiers et algorithmique. Thèse de doctorat soutenue à l'Université Louis Pasteur de Strasbourg, 1991.

R95. J.P. REveILLÈs, Combinatorial pieces in digital lines and planes. Vision Geometry IV, vol 2573 SPIE 1995.

RY97. J.P. REveILLÈs \& J. YAACOUB, Maximum area triangle operator for edge detection. Journal of electronic imaging 6(4), 406-414, 1997.

S97. J.M. Schramm, Coplanar Tricubes. 7th Conference on Discrete Geometry in Computer Imagery, Montpellier, 1997.

V99. L. VulLLon, Local configurations in discrete planes. Bull. Belg. Math. Soc. to be published 1999 . 